Ciencia y Educación, Vol. 4, No. 2, mayo-agosto, 2020

ISSN (impreso): 2613-8794・ ISSN (en línea): 2613-8808

DOI: https://doi.org/10.22206/cyed.2020.v4i2.pp87-102

\title{
Leer en la secundaria y en la universidad. Perspectivas de estudiantes de Psicología
}

\author{
Reading in high school and college. \\ Psychology Student Perspectives
}

\author{
María Micaela Villalonga Penna ${ }^{a}$ ORCID: 0000-0002-0211-6870 \\ Constanza Padilla ${ }^{\mathrm{b}}$ ORCID: 0000-0002-2213-5120
}

Recibido: 6/02/2020 Aprobado: 26/03/2020

Cómo citar: Villalonga Penna, M. M., \& Padilla, C. (2020). Leer en la secundaria y en la universidad. Perspectivas de estudiantes de Psicología. Ciencia y Educación, 4(2), 87-102. Doi: https://doi.org/10.22206/cyed.2020.v4i2.pp87-102

\section{Resumen}

El propósito de este estudio es describir las perspectivas que posee el estudiantado ingresante a la carrera de Psicología de una universidad argentina de gestión estatal, acerca de la lectura en la escolaridad secundaria y en contextos académicos. Se entrevista a 27 estudiantes del primer año de la mencionada carrera y se analizan estas entrevistas en función de categorías emergentes y que se desprenden del marco teórico. Los resultados muestran que los estudiantes con rendimiento académico alto perciben continuidades entre las prácticas lectoras del secundario y las de la universidad, y los que tienen un rendimiento bajo, diferencias sustantivas entre las mismas. Las continuidades y las diferencias se relacionan con las clases de textos y con las prácticas pedagógicas docentes. Estos hallazgos permiten entrever las diferencias derivadas de la escolaridad de base y la necesidad de abordar en las aulas universitarias la enseñanza de la lectura disciplinar en cada asignatura.

Palabras clave: lectura; estudiante; enseñanza secundaria; universidad.

\begin{abstract}
The purpose of this study is to describe the perspectives that the students entering the Psychology career of an Argentinian state-run university have about reading in secondary school and in academic contexts. 27 students from the first year of the aforementioned degree are interviewed and these interviews are analyzed according to emerging categories that emerge from the theoretical framework. The results show that students with high academic performance perceive continuities between high school and university reading practices, and those with low performance, substantive differences between them. Continuities and differences are related to the kinds of texts and to the pedagogical teaching practices. These findings allow us to glimpse the differences derived from basic schooling and the need to address the teaching of disciplinary reading in each subject in university classrooms.
\end{abstract}

Keywords: Reading; student; secondary school; college.

\footnotetext{
a Instituto de Investigaciones sobre el Lenguaje y la Cultura (INVELEC) del Consejo Nacional de Investigaciones Científicas y Técnicas (CONICET), Facultad de Psicología, Universidad Nacional de Tucumán, Argentina Correo-e: micaelavillalongapenna@conicet.gov.ar

b Instituto de Investigaciones sobre el Lenguaje y la Cultura (INVELEC) del Consejo Nacional de Investigaciones Científicas y Técnicas (CONICET), Facultad de Filosofía y Letras, Universidad Nacional de Tucumán, Argentina Correo-e: constanzapadilla@conicet.gov.ar
} 


\section{Introducción}

En esta investigación se parte de un interrogante general ¡cómo concibe las prácticas lectoras académicas el estudiantado del primer año de Psicología? En relación con este interrogante de investigación, se indaga también si perciben diferencias entre las prácticas lectoras del secundario y las de esta comunidad disciplinar y con qué aspectos se vinculan las diferencias percibidas. Se plantea esta pregunta ya que desde los enfoques teóricos de las alfabetizaciones académicas y de la literacidad crítica, diversos estudios han dado a conocer la distancia percibida por los estudiantes entre las prácticas lectoras propias del nivel secundario y las de la universidad. Al entrar en la universidad los estudiantes deben apropiarse de una nueva cultura, la académica, y esto no depende solo de la comprensión explícita de las materias enseñadas, sino también del aprendizaje de prácticas académicas que poseen aspectos explícitos e implícitos - por ejemplo, la comprensión implícita de cómo satisfacer las expectativas docentes- (Collier y Morgan, 2008). En palabras de Hermerschmidt (1999, p. 6): "la entrada en la universidad es un escalón en una institución que tiene una serie de expectativas que deben ser reconocidas por los recién llegados".

Por su parte, Theresa Lillis (1999) describe una serie de desencuentros entre las expectativas del profesorado y las interpretaciones del estudiantado, sobre lo que implica leer y escribir. Cuando los profesores plantean sus consignas de lectura y de escritura, lo que para ellos parecen ser pedidos explícitos y suficientemente claros, para los estudiantes puede resultar impreciso, ambiguo y contradictorio. Esta autora propone que para el estudiantado lo evidente resulta extraño e idiosincrático y esto puede llevar a que no comprendan cómo son valorados sus trabajos y a que perciban sus conquistas o fracasos como el corolario de haber podido o no ajustarse a demandas arbitrarias (Lillis, 1999). Entonces, bajo estas condiciones, las formas esperadas de leer y escribir en la universidad pasan a ser un enigma. (Lea y Street, 1998).

Lillis (1999) considera que las prácticas de lectura y de escritura en el nivel superior y la comprensión de las normas y formas que estas adoptan, suelen ser vistas como simples, no problemáticas y vinculadas a conocimientos que los estudiantes debieran haber construido en el nivel educativo anterior. Sin embargo, "cuando las formas que adoptan estas actividades no son comprendidas por aquellos menos familiarizados con las convenciones de la lectura y de la escritura académicas, las mismas se transforman en "prácticas institucionales del misterio” (Lillis, 1999, p. 127).

Otra faceta de las distancias entre las prácticas lectoras del secundario y las de la universidad es la abordada por Collier (2001), quien señala que, si bien un estudiante en el nivel educativo previo puede haberse percibido como un lector y escritor muy capaz, al alcanzar un nivel educativo superior puede sentirse poco habilitado para leer y escribir, y para comprender las demandas y adecuarse a las exigencias, corriendo el riesgo de desalentarse e incluso frustrarse (Lea y Street, 1998). Es así que, las experiencias lectoras construidas en el nivel secundario, pueden facilitar u obstaculizar el aprendizaje de las prácticas lectoras disciplinares académicas (Collier y Morgan, 2008).

Desde estos enfoques teóricos, se cuestionan las condiciones de acceso y de permanencia en la educación superior, porque no basta con que el alumnado asista a clase, sino que es necesario que pueda significar y participar de forma reflexiva y consciente en las prácticas alfabetizadoras - especialmente las de lectura y de escritura - de este nivel (Carlino, 2019a; Carlino, 2019b). La participación reflexiva y activa tiene que ver con la construcción de conocimientos acerca de una serie de elementos, entre los que podemos mencionar, los géneros discursivos o las clases de textos que se leen en la universidad y las diferentes formas de organización que adoptan las prácticas lectoras. Cabe señalar que estas son cuestiones que ampliaremos en el apartado "Referente Teórico".

En este sentido, el propósito de esta investigación es describir las perspectivas acerca de la lectura en la escolaridad secundaria y en contextos universitarios, que poseen los estudiantes ingresantes a la carrera de Psicología de una universidad argentina de gestión estatal. 


\section{Referente teórico}

Algunos de los estudios revisados se centraron en las concepciones sobre las prácticas lectoras en el secundario y en la universidad (Carlino, 2019a; Carlino, 2019b; Fernández y Carlino, 2010). Al respecto, Fernández y Carlino (2010) indagaron las perspectivas sobre las prácticas de lectura académica del alumnado de los primeros años de carreras de dos áreas: Ciencias Humanas y Veterinarias, ambas de una universidad argentina de gestión pública. Los alumnos reconocieron diferencias entre los textos que leían en el secundario y los que leen en la universidad. Los textos del área de Ciencias Humanas eran complejos porque incluían referencias de diferentes autores a veces contrapuestas y un conocimiento relativo - lo cual no sucedía en el secundario- y los de Ciencias Veterinarias, presentaban el conocimiento como acabado, universalizable y único. Leer en ambos campos disciplinares implicaba aprender el discurso especializado de cada uno de ellos. Además, en Ciencias Humanas la lectura exigía aprender a relacionar los textos y los autores entre sí, y leer varias veces los textos y comprenderlos desde la perspectiva de una cátedra y de una comunidad disciplinar. Para las autoras, esta investigación mostraba cómo los ingresantes a la universidad deben aprender la cultura académica, la cual resulta muy distinta a la cultura de la cual provienen. También daba a conocer que las dificultades lectoras tienen que ver muchas veces con falta de experiencia de los recién llegados con los modos de lectura que se piden en la universidad (Fernández y Carlino, 2010).

La bibliografía consultada dio a conocer que en la universidad suelen leerse textos académicos derivados de textos científicos — por ejemplo, capítulos de libros elaborados por referentes de la disciplina o capítulos de manuales introductorios a disciplinasy textos científicos - por ejemplo, trabajos de investigación, artículos de revistas especializadas- (Bigi, Chacón y García, 2013; Carlino, 2019a; Giudice, Godoy y Moyano, 2016; Godoy y Giudice, 2015; Villalonga Penna, 2015; Villalonga Penna y Padilla, 2016; Villalonga Penna y Padilla, 2019). En cuanto a las formas de acceso al material, los estudiantes suelen leer fotocopias de textos (Carlino, 2019a; Giudice, Godoy y Moyano, 2016; Villalonga Penna, 2015).

Otro grupo de desarrollos tuvo su eje en el tipo discursivo (Arnoux, Di Stefano y Pereira, 2002; Padilla, Douglas y López, 2015; Villalonga Penna, Padilla, Carreras y Carreras, 2019; Villalonga Penna y Hael, 2016) o función textual (Heinemann y Viehweger, 1991) predominante de los textos a los cuales acceden los estudiantes en la universidad. Básicamente, en medios académicos suele apreciarse la presencia de textos expositivos y argumentativos. A este respecto, Arnoux et al., (2002) entienden a "la exposición y a la argumentación como dos polos de un continuum, por lo cual, los discursos pueden tender al polo expositivo-explicativo o al argumentativo" (p. 1). En la misma dirección, Padilla, Douglas y López (2007, 2011) plantearon que los textos pueden ser predominantemente expositivos o argumentativos. Los primeros, o que tienden al polo expositivo-explicativo, se centran en un saber construido y socialmente legitimado, buscan informar y tienden a borrar las huellas subjetivas para marcar una distancia y objetividad. Suelen apelar a citas de autoridad. Los textos predominantemente argumentativos o que tienden al polo argumentativo, buscan persuadir y el interjuego entre múltiples voces $\mathrm{u}$ opiniones procura construir nuevos conceptos o posturas (Arnoux et al., 2002; Padilla et al., 2007 y 2011).

Asimismo, una serie de investigaciones han indagado cómo se configuran las prácticas de lectura y escritura en contextos áulicos en diferentes niveles educativos, entre ellos, el superior universitario y no universitario. Retomamos estos aportes ya que, si bien no tienen su eje puesto en las concepciones o perspectivas de los actores educativos respecto de las mencionadas prácticas, sus resultados pueden ser de utilidad para comprender algunos de los fenómenos descritos en nuestra investigación por los entrevistados. Así, los estudios internacionales (Dysthe, 1996; Wells, 2006) y nacionales (Cartolari y Carlino, 2012; Cartolari y Carlino, 2016; Padilla, 2017) han dado a conocer dos formas de organización de las prácticas lectoras áulicas: las dialógicas y/o monológicas. 
Las formas monológicas, se caracterizan por un predominio de las exposiciones orales por parte del docente y por la concreción de comunicaciones organizadas por turnos determinados por este. Estas interacciones comunicacionales al estar determinadas y autorizadas por los docentes, suelen no propiciar que los estudiantes realicen contribuciones significativas al tema trabajado (Cartolari y Carlino, 2012; Cartolari y Carlino, 2016; Carlino, Cordero Carpio, Roni y Rosli, 2017; Dysthe, 1996; Wells, 2006). Muchas veces, los docentes hasta pueden llegar a evitar las interacciones verbales sobre lo leído o lo escrito por estimarlas como instancias poco productivas y/o como una pérdida de tiempo (Dysthe, Samara y Westrheim, 2006). Se trata de situaciones en las que las explicaciones del profesorado terminan siendo la fuente principal de información y "la bibliografía no es un medio para construir interpretaciones y apropiarse de los modos de decir en la disciplina de formación" (Cartolari y Carlino, 2012, p. 81).

Esta forma de organización de las prácticas de lectura se relaciona con lo que se ha denominado prácticas de lectura en los extremos o en los márgenes exteriores (Carlino, Iglesia y Laxalt, 2013) en las que el docente daba una consigna, los estudiantes la resolvían sin la intervención del docente y luego este evaluaba lo producido, señalando los errores o falencias de los alumnos y alumnas al leer, para erradicarlos. Lo que sucedía durante las actividades de lectura era responsabilidad de los alumnos y las indicaciones y el feedback del docente ante inquietudes, no eran vistos como necesarios. En este punto, la revisión de lo leído solo se hacía para subsanar estos errores - y no como una actividad conjunta que permite construir conocimientos de la asignatura- (Carlino et al., 2013).

Las formas dialógicas implican un predominio de las interacciones entre el profesorado y el alumnado, basadas en preguntas que no se encuentran predefinidas y que enfocan la lectura durante las clases. Además, el profesorado da lugar a las conversaciones acerca de lo leído y ofrece pautas orientativas durante las mismas. Lo más importante parece ser la generación de una intertextualidad, los intercambios orales en el aula y el discurso escrito presente en la bibliografía. En este sentido, la interrelación entre lectura, escritura e intercambios orales en las clases sirve para potenciar los aprendizajes en dos direcciones: leer y escribir para hablar, y hablar para leer y escribir (Dysthe, 1996). Son interacciones en el aula en torno a los textos disciplinares que permitirían el acceso del estudiantado a las formas de leer y comprender en disciplinas específicas (Carlino 2017a; Carlino, 2017b; Carlino 2018a; Carlino, Cordero Carpio, Roni, Rosli, 2017; Cartolari y Carlino, 2012; Cartolari y Carlino, 2016; Dysthe, 1996; Wells, 2006).

Esta manera de organizar las prácticas lectoras se vincula con lo que se ha llamado prácticas de lectura entrelazadas (Carlino, 2017; Carlino, 2018a; Carlino 2018b; Cartolari, Carlino y Laxalt, 2013) o prácticas lectoras conjuntas (Braidot, Moyano, Natale y Roitter, 2012; Natale y Stagnaro, 2013) en las que los docentes proponen mediar los procesos lectores durante las clases e incentivar las interacciones para hablar sobre lo leído entre docentes y alumnos y entre pares. Quienes intervienen durante la tarea, lo hacen para ayudar al estudiantado en la realización de la misma, observar cómo resuelven las actividades y qué dificultades tienen durante el proceso - y no solamente en los productos-. En este caso, la lectura es un medio para trabajar los temas de la materia - es decir, que las tareas de lectura se entrelazan con el aprendizaje de contenidos conceptuales_- El profesorado promueve las interacciones alumnos- docente, buscando que haya un ida y vuelta recursivo entre lo que hacen tanto los alumnos como las alumnas y, tiempo a la lectura y relectura conjunta con los alumnos (Carlino 2018a; Carlino, 2018b; Carlino et al., 2013).

\section{Metodología}

Se optó por un abordaje metodológico cualitativo, ya que buscamos conocer los significados que el estudiantado asigna a sus experiencias como lectores académicos. El muestreo fue de tipo teórico en tanto los datos recolectados y la teoría emergente guiaron la conformación de la muestra (Hernández Sampieri, Fernández Collado y Baptista Lucio, 2008).

De la totalidad del estudiantado del primer año de la carrera de Psicología, en este estudio, participaron estudiantes que habían obtenido calificaciones altas 
(entre $10 / 10$ y $6 / 10$ puntos) y bajas (entre $5 / 10$ y $2 / 10$ puntos) en las primeras evaluaciones parciales de las materias. De los 27 (veintisiete) estudiantes que participaron, 17 (diecisiete) eran de sexo femenino y 10 (diez), de sexo masculino. La media de edades era de 18 (dieciocho) años. Cabe señalar que antes de comenzar el estudio se obtuvo el consentimiento informado de los participantes.

Se obtuvieron los datos apelando a entrevistas en profundidad a estudiantes. Se entrevistó a 27 (veintisiete) estudiantes dos veces durante 60 minutos promedio, a dos meses y medio del inicio de clases. Los estudiantes cursaban 6 (seis) asignaturas diferentes y se les preguntó acerca de sus concepciones en relación con la lectura académica. Los alumnos fueron seleccionados teniendo en cuenta los puntajes obtenidos en el primer examen o evaluación parcial (correspondiente al primer semestre) de la asignatura seleccionada: 14 con baja puntuación ( 2 a 5 puntos $/ 10$ puntos o alumnos desaprobados) y 13 con alta ( 6 a 10 puntos/10 puntos o alumnos aprobados). A fin de preservar la confidencialidad de la identidad de los participantes en este estudio se ha denominado a los estudiantes del 1 (uno) al 27 (veintisiete). Además, los enfoques y herramientas metodológicas cumplen con los procedimientos éticos establecidos para la investigación científica en humanidades.

Para este estudio de corte exploratorio y descriptivo, se siguieron los lineamientos de la teoría fundamentada de tipo emergente — con lo cual a partir de los datos, se construyó una teoría que diera cuenta del fenómeno- - Luego de realizar las primeras entrevistas a los estudiantes, se analizaron buscando categorías preliminares de manera inferencial. A partir de estos datos, se conformaron los grupos de estudiantes con calificaciones altas y bajas. Posteriormente, se realizaron las segundas entrevistas, depurando de forma progresiva la búsqueda de categorías hasta llegar a la saturación. Esto se logró a partir de la comparación constante que posibilitó identificar lo que resultaba repetitivo en el discurso estudiantil. En último lugar, se generó supuestos relacionados entre sí que posibilitaran contar con una explicación del fenómeno estudiado (Hernández Sampieri et al., 2000). Se efectuó el análisis con el apoyo del Atlas.ti versión 6.0.15.

\section{Resultados}

Del análisis de las entrevistas a los estudiantes se desprenden dos grandes categorías que hemos denominado "Leer en el secundario y leer en la universidad "y "Leer en el secundari o aleer en la universidad". A su vez, estos temas comprenden una serie de categorías referidas a las clases de textos y los tipos discursivos de los textos que declaran leer estos estudiantes y a cómo conciben las prácticas lectoras que se realizan en esta comunidad. El primer tema abarca las perspectivas de los estudiantes que han tenido calificaciones altas y el segundo, las de los estudiantes que han tenido calificaciones bajas.

\subsection{Leer en el secundario y leer en la universidad}

Los estudiantes que han tenido calificaciones altas perciben continuidades entre las prácticas lectoras del secundario y las de la universidad, vinculadas con el tipo de textos y, en algunos casos, con las prácticas de enseñanza de la lectura.

En función de estos hallazgos se indagó en torno a las clases de textos que se leían en el secundario y en la universidad. Estos estudiantes han trabajado con manuales y textos introductorios universitarios durante la secundaria.

E: ¿Vos notás diferencias entre los textos que leías en el secundario y los que leés ahora?

A: Y... en el cole nos daban algunos cuadernos introductorios de materias de la universidad para estudiar y también los manuales... pero la cantidad a leer nunca era tanto como aquí... pero hay textos como parecidos a los que leíamos en el colegio (alumno 2, G1).

E: ¿Y cómo era leer en la secundaria y leer ahora en la universidad?

A: En el cole leíamos manuales y aquí también... los textos son más largos. A veces en el cole leíamos capítulos de libros como los que se leen en la universidad porque los profes

a. La Educación Secundaria en la provincia de Tucumán (Argentina), Según la Ley Nacional de Educación $N^{\circ}$ 26206, tiene una duración teórica de 6 años y las edades teóricas de comienzo y finalización son 11 y $17 / 18$ ańos, respectivamente. 
querían que vayamos entrando en contacto con este tipo de textos, pero siempre nos daban una presentación teórica y guías de estudio para facilitarnos la lectura. Pero bueno, aquí en la facu leemos estos textos difíciles, sobre todo esos textos originales de los autores.

$\mathrm{E}:$ ¿Y por qué te parecen difíciles?

A: Son largos, te nombran gente, teorías que no conocés, dan vueltas y te llenan de ejemplos, dicen una cosa, después la niegan, y después viene lo que dice el autor que a todo esto, viene criticando a otros fulanos que ni conocés y que hablan de cosas que tampoco sabés, y cuando llega a una conclusión a veces no es tan cantada. En el cole nos enseñaron que eso es argumentar. Te marean, te despistan y tratan de convencerte o de engañarte...si...si... Vimos algunos textos así en el cole y otros que explican nada más (alumno 8, G1).

A: Es raro, hay materias en las que... los textos son más largos y más difíciles que los que leíamos en el cole, pero haciendo lo que aprendí en el cole puedo leerlos y entenderlos.

E: ¿Cómo vendría a ser eso, podés explicarme más?

A: En el cole en casi todas las materias nos hacían leer los textos una o dos veces y buscar ideas principales. Vos podías tener una clase de guía que daba el profe antes o después de leer el texto. Nos enseñaron a hacer esquemas con la estructura del texto y los conceptos más importantes y había que saber quién escribía, el ańo, la edición... a veces leíamos los manuales, otras veces novelas, cuentos, capítulos de libros que se leen en la facu, artículos de ciencia... A veces había que comparar lo que decían varios autores sobre un mismo tema, hacíamos cuadritos comparativos... y después llego aquí $y$ algunos profes me dicen que lo que se dio en el teórico eso busquemos en los textos y otros te hacen estudiar más como me enseñaron en el cole (alumno 13, G1).

Solamente los estudiantes con calificaciones altas habían recibido instrucción acerca de tipos discursivos durante la escolaridad secundaria y señalan que en la bibliografía de las materias hay textos que exponen y textos que argumentan.

E: ¿Vos notás diferencias entre los textos de las materias?

A: Y sí... porque hay textos que son de esos que argumentan y hay otros que son de esos que explican... los de Problemas ${ }^{\mathrm{b}}$ y los de Introducción ${ }^{c}$ son como que argumentan, hay muchos autores que opinan dentro del texto y se contradicen... y los Psico $^{\mathrm{d}}$ o la Integración ${ }^{\mathrm{e}}$ o Vital ${ }^{\mathrm{f}}$ como que exponen porque son como que te dicen las cosas sin vueltas.

$\mathrm{E}:$ ¿Dónde has aprendido eso?

A: En el cole, la profe de lengua nos hacía estudiar eso (alumno 1, G1).

Los estudiantes diferencian dos formas de lectura: una, que se centra en la identificación de información específica en los textos y la otra, que acentúa la relación entre lo propuesto por autores en diferentes textos, las explicaciones de los docentes y contextuar lo que se lee. Mientras que valoran la segunda forma de enseñanza de manera positiva, la incongruencia percibida entre ambas formas de lectura genera incertidumbre.

E: ¿Los docentes te orientan acerca de cómo leer los textos de las materias?

A: En Problemas y en Antropología sí... en las otras no... ponele, en Psicofisiología, en Vital y en Intro te hablan de los conceptos y de los conceptos que hay que saber pero no en qué texto están, y por qué un autor dice lo que dice... es raro, es como que se pierde eso... en cambio, en Problemas te dicen que veas bien que viene diciendo el autor antes y después, que hagas esquemas sobre cómo va presentando los temas el autor, que sepás quién es el autor, en qué momento escribe... la profe muchas veces lee junto con nosotros y está con el texto... no con la cosa aislada

E: ¿Qué cosa?

A: Don concepto (risas)

\footnotetext{
b. Problemas Sociológicos en Psicología

c. Introducción a la Psicología

d. Psicofisiología

e. Integración Universitaria

f. Psicología del Curso Vital I
} 
E: Vos me decís entonces, que hay como dos formas de leer y que varía según las materias ¿sí?

A: Sí

E: Y que no tenés dificultades para leer, pero que estas dos formas de lectura y en qué materia resultan apropiadas, te confunde

A: Sí, esa sería la dificultad o el problema que tengo

E: ¿Los docentes te ayudan con esto?

A: No mucho la verdad... ellos te dicen qué hacer para aprobar... pero no te hablan de leer... los que te dicen qué cosas buscar en los textos no te hablan de leer... te hablan de buscar las cosas en los textos... los que te hablan de leer los textos en diferentes etapas, viendo quién escribe, las ideas principales, el esquema del texto y todo eso, esos profes son los que hablan de leer los textos. El tema es averiguar cómo leer en cada materia. Pero sigo confundido...

E: ¿Qué es lo que te confunde?

A: Pasa que en el colegio nos enseñaron a leer los capítulos de los manuales o de los libros introductorios de la universidad y nos hacían ver eso de quién es el autor, en qué año se escribió el texto y nos daban preguntas que vos podías encontrarlas con facilidad en el texto o que había que leer varias veces el texto o dos textos para poder contestarlas... o ver los apuntes que habíamos tomado en la clase para completar las preguntas... entonces esto de leer y buscar solo lo que el profesor dice que te hace aprobar es como distinto a lo que me han enseñado en el cole (alumno 3, G1).

A: ...Y está esto de leer y entender lo que el profesor quiere, eso de buscar los conceptos o las cosas en el texto y está lo otro de leer y tratar de ir viendo que dice el autor y cómo se relaciona eso del autor con otros autores. Y son como distintas cosas ¿no? Pero me confunde que me pidan esas dos formas de leer en las materias ¿cuál es la correcta? Porque si leo de una forma en una materia no me sirve y si leo de otra forma en otra materia tampoco me sirve E: ¿Los docentes te ayudan con esto?
A: No mucho la verdad... ellos te dicen qué hacer para aprobar... pero no te hablan de leer... los que te dicen qué cosas buscar en los textos no te hablan de leer... te hablan de buscar las cosas en los textos... los que te hablan de leer los textos en diferentes etapas, viendo quién escribe, las ideas principales, el esquema del texto y todo eso, esos profes son los que hablan de leer los textos. El tema es averiguar cómo leer en cada materia. Pero no entiendo... porque a mí en la escuela me habían enseñado a resumir o responder las preguntas viendo distintos textos y los apuntes de la clase y a complementar ¿̨no? Y ver el autor y en qué año ha escrito y ver si es capítulo de libro o ficha de estudio o capítulo de un libro de catedra de la universidad... y las preguntas que nos daban no eran de esas cantadas que contestas con un párrafo del texto... bueno, podía haber de esas, pero no eran todas... había otras que se contestaban haciendo eso que te digo de complementar textos o con diferentes partes del mismo texto (alumno 6, G1).

\subsection{Leer en el secundario o leer en la universidad}

En relación con los estudiantes que han tenido un rendimiento bajo, señalan una marcada brecha entre las prácticas lectoras propias del secundario y las de la universidad. Esta distancia se centra en la cantidad a leer, en el tipo de guías de lectura a resolver y en la clase de textos a leer.

A: ¡Ufff? Sí, es un montón para leer, las fotocopias, son muy largas, no son dos o tres hojitas de un manual, son partes enteras de libros... es la cantidad que hay que leer ...y las guías que nos dan, en los prácticos, es como que nos dan una pregunta a cada grupo y la contestás... con el texto y después se revisa entre todos... vos tenés una pregunta pero no todas y hay que resolverlas en la casa o dividir entre los compañeros del grupo (alumno 17, G 2).

A: ¿Diferencias? Eso es poco. Es otra cosa, es una banda. Nada que ver lo que hacíamos en la escuela con esto. 
E: ¿En qué sentido?

A: No es lo mismo que te dicten una guía con... las preguntas y las respuestas... que leer textos, enteros, larguísimos, eternos. A veces consigo resúmenes y estudio de ahí, es más fácil... es que los textos de Psicofisiología son llenos de detalles... y los resúmenes me ayudaron a llegar al parcial. Y los de Introducción son re difíciles, como los de Problemas. Te hacés un lío, porque son largos y complicados.

E: ¿Por qué decís que son complicados?

A: Porque son largos, con letra re chiquita

y es como que te perdés cuando los leés. Son llenos de detalles, palabras nuevas, nombran un montón de gente que ni sé quiénes son. En Introducción tenés el libro que te dice las cosas en fácil, pero en Problemas no tenés un librito así.

E: Entonces, hay textos que nombran mucha gente, y que tienen detalles ¿y qué los hace distintos de los textos de Psicofisiología, por ejemplo?

A: Los de Psicofisiología también tienen muchas palabras nuevas pero no te nombran gente nueva que dice cosas que no sé, como que esos textos de Problemas o Introducción se hacen eternos por la cantidad de gente que dice cosas.

E: Y esta gente, que dice cosas ¿esas cosas, tienen algo que ver con lo que dice el autor del texto?

A: Yo calculo que sí, pero es que de esos textos en Introducción me pasaron resúmenes y Problemas la dejé. Ya me pasaron unos resúmenes así que la voy a hacer el año que viene (alumno 10, G 2).

A: Nada que ver una cosa con otra porque no hay cuadernillo con los resúmenes hechos por los profes, salvo, lo más parecido son las fichitas esas de las cátedras... es como difícil leer textos tan largos y saber que te van a tomar aunque vayas a las clases teóricas. Y los profes nos dan preguntas para responder pero algunas las podés contestar con los apuntes de la teórica, otras con los resúmenes que me pasaron mis compañeros y la verdad que no sé si se responden con las fotocopias porque leí muy pocas (alumno 15, G 2).

E: ¿Y de qué eran las fotocopias que te daban en la escuela?

A: No sé bien, creo que de manuales... y bueno esa fotocopia se leía (alumno 5, G 2).

A: Guías con las respuestas... Alguna vez un profe de historia nos hizo sacar copias de un manual y subrayar las ideas principales después de que nos explicó el tema y responder la guía de preguntas... Porque en el cole sabíamos que los profes querían que aprobemos. Sabíamos qué había que estudiar las guías y que con eso se aprobaba (alumno 10, G 2).

Resulta llamativo también que estos alumnos se refieran a los textos como fotocopias, de las cuales en muchos casos desconocían su origen o suponían, sin poder asegurarlo, que los docentes las habían preparado. Además, cuando el origen de las fotocopias era explicitado, parecía no ser algo sustancial para los alumnos.

A: ...en la escuela eran cortitas las fotocopias y los profes nos dictaban las guías o los temas, como si nos dictaran lo que había que saber.

E: Las fotocopias que les daban en la escuela ¿de dónde venían?

A: De los profes

E: Me refería a si venían de libros o revistas o manuales.

A: No sé, ni idea. Eran fotocopias.

E: ¿De alguna manera sabían si esas fotocopias tenían un autor o estaban incluidas en un libro? A: No, eran fotocopias, no sé más que eso (alumnos 13, G 2).

E: Y esas fotocopias que sacaban ¿de dónde venían?

A: No sé, ni idea

E: ¿Eran fotocopias de libros?, ¿de alguna forma se indicaba si eran fotocopias de un manual, suponete, o se indicaba el autor?

$\mathrm{A}$ : $\mathrm{Si}$, algunas veces los profes nos decían es de tal manual, pero nos decían, así, de palabra, y otras veces eran resúmenes de los temas 
que calculo que hacían los profes, pero bueno, supongo que los profes eran los que la habían escrito (alumno 14, G 2).

Solamente estos estudiantes declaran que utilizan como material de estudio fotocopias de resúmenes y de carpetas que no constituyen una elaboración personal. Respecto de quién ha elaborado la carpeta o el resumen, si bien las emplean para estudiar, no saben cuál es su procedencia. La manera de evaluar cuán válidos y confiables resultan estos textos, se relaciona con la capacidad de aprobar exámenes parciales con base en los mismos.

A: Es más fácil... están las carpetas que les sacas fotocopia... son como resúmenes de los cuadernillos.

$\mathrm{E}:$ ¿Y vos sabés quién ha hecho esos resúmenes? A: No, ni idea, la verdad que no, pero nos fue bien a todos los que estudiamos de ahí para el parcial, entonces tan mal no deben estar (alumno 23, G 2).

Además, indican como aspectos diferenciales entre los textos que leían en el secundario y los que leen en la universidad, el tamaño de la letra — chiquitay la inclusión o no de paratextos — dibujos-.

A: Las cosas que leemos, textos largos, enteros, largos y con letra chica y sin dibujos... Psicofisiología traté de leer los textos pero son largos y con letra re chiquita y dibujos que dicen las profes que hay que estudiar también y bueno, me pasaron una carpeta que tiene todo resumido y así zafé con el primer parcial (alumno 11, G 2).

E: De las materias, ¿los textos de qué materias te parecen más complejos o difíciles?

A: Y los de Psicofisiología que hablan de cosas que casi no vi en la escuela y son larguísimos, con letras chiquitas y esos dibujos (alumno $14, \mathrm{G} 2)$.

Expresan que en la universidad leen cuadernillos y fotocopias de libros sin poder acceder a los originales y/o sin poder precisar su origen. Cabe señalar, que algunos de estos estudiantes no mencionan la lectura de textos, sino de fotocopias. Además, notan la presencia de palabras nuevas y en otros idiomas en los textos.
E: ¿Y qué leés aquí en la facultad?

A: Mmmm...fotocopias (se ríe)

$\mathrm{E}$ : Bien, pero fotocopias ¿de qué?

A: $Y$ de los apuntes que hay que estudiar

E: Claro, pero, de esas fotocopias, ¿les explicaron de dónde vienen?

A: Sí, de los libros, pero a los libros yo no los veo en vivo y en directo, porque está la fotocopia.

E: ¿Las fotocopias siempre son de libros?

A: Supongo, no sé, yo sé que está el cuadernillo con los apuntes y que de ahí hay que leer (Alumno 5).

E: Está bien. Y... ¿qué... qué hacen con el material que tienen para leer?

A: Como en casi todas las materias tenés esos cuadernillos con las fotocopias de lo que hay que leer (alumno, 16, G 2).

E: ¿Qué hacés cuando leés un texto?

Plantean la distinción de textos — apuntes o fotocopias - en función de la longitud. Además, señalan que los textos cortos suelen ser más fáciles y entendibles, y los largos, más complicados, con letra chiquita, eternos.

A: Hay cosas que leemos que son más accesibles. $\mathrm{E}$ : ¿Accesibles en qué sentido?

A: No son largos los apuntes, las fotocopias, y son de cosas que más menos se entienden... eso lo de Antropología, y la Integración, eran apuntes... eran más cortos y más fáciles, más accesibles, entonces, se entienden bien cuando uno lee...

E: Entonces ¿hay textos que son fáciles y otros que no?

A: Claro

$\mathrm{E}:$ ¿Y cómo son los textos fáciles?

A: Cortos

E: O sea, los textos fáciles son los cortos.

A: Sí, y además son fotocopias que los conceptos importantes se encuentran más fácil (alumno5, G 2).

E: ¿Por qué decís que son complicados?

A: Porque son largos y complicados... y con letra re chiquita y hay que leer esos textos eternos, eternos (alumno 10, G 2). 
Estos estudiantes solo describen prácticas lectoras basadas en guías de estudio tanto en el secundario como en la universidad. Mientras que en el secundario muchas veces las guías ya estaban resueltas o se respondían en la casa con base en textos cortos o resúmenes hechos por los docentes, en la universidad las guías se resuelven en clase - solo en parte- con base en los apuntes de la clase teórica, la bibliografía contenida en los cuadernillos o resúmenes hechos por terceros. Valoran negativamente la presencia de múltiples fuentes textuales.

E: ¿Y qué diferencias notás entre lo que hacían al leer en el secundario y lo que hacen aquí?

A: En la escuela era mejor, era más fácil. Los profes te dictaban las guías con las preguntas y las respuestas o te daban guías para hacer en la casa y las respuestas estaban fáciles de encontrar en los apuntes o en los manuales... los resúmenes que nos daban los profes de los temas y eran pocas hojitas del manual... y aquí las fotocopias son eternas y para responder a las guías se hace desde varias fotocopias, muy complicado o hay preguntas que se contestan con diferentes partes de una fotocopia... muy complicado (alumno 12, G2).

E: ¿Qué hacían al leer en el secundario y qué diferencias ves con lo que haces ahora?

A: En la escuela nos daban los resúmenes para leer y unas preguntas que las encontrabas fácil en los textos... aquí las preguntas no se encuentran fácil y es un montón para leer y poder contestar una nomás... y además en las clases prácticas el profe llega le da a cada grupito una pregunta de la guía, la contestás con los apuntes de la teórica, o con resúmenes que fotocopiamos o con los cuadernillos.

E: ¿Y qué hace el docente mientras ustedes trabajan?

A: Nada...

E: Y si ustedes lo llaman para consultarle algo.

A: Se acerca pero no te dice mucho... pero no hacen nada en general, y después exponemos lo que hemos hecho, el profe te dice si está bien o mal y listo. Una vez una profe nos dijo que ya teníamos que trabajar solos... que ya no estamos en la escuela... que contestemos las preguntas con la biblio y que después veíamos cómo nos había ido con la respuesta en la puesta en común.

$\mathrm{E}:$ ¿Y de qué otra forma trabajan en las clases?

A:Y... siempre hacemos así... como te contaba... siempre hacemos así... y lo que pasa es que a veces lo que contestamos no le parece bien a los profes y entonces ellos te dicen, pero nunca termino de saber bien qué hacer porque te dice que está incompleto y te nombra detalles y nunca sé bien en qué fotocopias o en qué parte de las fotocopias están esos detalles... esas cosas que faltan completar... como que no sé qué es lo que quieren los profes...(alumno 14, G2).

\section{Discusión}

Estos hallazgos dan a conocer que solo el estudiantado con calificaciones altas parece reconocer características acerca de cómo están escritos los textos (Carlino, 2019a; Villalonga Penna, y Padilla, 2016) o los tipos discursivos (Arnoux et al., 2002; Padilla et al., 2007 y 2011; Villalonga Penna y Padilla, 2016). Al igual que lo hallado por Fernández y Carlino (2010), la lectura se ve facilitada cuando los textos se parecen más a los leídos durante el secundario. Como lo plantean estudios locales (Villalonga Penna, 2015; Villalonga Penna et al., 2019) vemos que la gran mayoría de los estudiantes no poseen sólidos conocimientos sobre la dimensión comunicativa de los textos, especialmente la argumentativa. Sin embargo, el estudiantado con alto rendimiento pareciera identificar más detalles en torno a esta cuestión e inclusive la conceptualizan. Por su parte, los estudiantes con un bajo rendimiento, parecen quedarse con aspectos superficiales como el tamaño de la letra, la presencia o ausencia de paratextos, la longitud de los textos y el léxico novedoso (Fernández y Carlino, 2010).

En concordancia con los hallazgos de estudios nacionales (Fernández y Carlino, 2010) e internacionales (Collier y Morgan, 2008; Lea y Street, 1998) se aprecia cómo se genera una distancia entre el secundario y la universidad, y cómo la experiencia lectora del secundario marca una diferencia entre los estudiantes. Tal 
como lo plantean estudios nacionales, vemos que el alumnado que tuvo un buen rendimiento, durante su escolaridad secundaria ha accedido a una variedad de textos, y entre ellos, textos académicos (Padilla et al., 2007, 2011 y 2015). En cambio, el alumnado que tiene un bajo rendimiento ha accedido, al igual que lo seńalado por Carlino (2019a) y por Villalonga Penna y Padilla (2016), a fotocopias cuyo origen era desconocido o no parecía ser significativo para los estudiantes y a lo que llamamos textos "simplificados" - guías con preguntas y respuestas o dictado del tema a estudiar-. Además, a diferencia de la educación universitaria, en la secundaria, utilizaban textos con un bajo nivel de complejidad y una única fuente textual (Fernández y Carlino, 2010; Padilla, 2017).

En la misma dirección que teorizaciones internacionales realizadas desde las Alfabetizaciones Académicas (Collier y Morgan, 2008), la institución escolar (Fernández y Carlino, 2010) marcaría diferencias sustantivas en la apropiación de la cultura lectora académica —algunos habrían aprendido formas de lectura en el nivel secundario que les permitían hacer frente a los desafíos académicos de manera más autónoma y otros no-.

El estudiantado con calificaciones altas reconoce la lectura de fotocopias de capítulos o partes de libros y fichas de cátedra, lo cual resulta afín con hallazgos nacionales (Carlino, 2019; Fernández y Carlino, 2010; Villalonga Penna y Padilla, 2016) e internacionales (Bigi et al., 2013). Por su parte, el alumnado con calificaciones medias y bajas, menciona la lectura de resúmenes y carpetas que no constituyen una elaboración personal y cuyas procedencia y autorías resultan desconocidas, los cuales, como clases textuales, no han sido descritos en la literatura de especialidad consultada. Como ya se ha mencionado, el alumnado con calificaciones medias y bajas se refiere a los textos como "cuadernillos" y "fotocopias" y el alumnado con calificaciones altas, habla de textos y fotocopias de textos, lo cual resulta afín a lo propuesto por Carlino (2019) y por Iglesia y de Micheli (2009).

Tal y como lo plantean aportes nacionales (Fernández y Carlino, 2010; Padilla et al., 2015), el estudiantado de los dos grupos remarca la gran cantidad a leer para cada materia y la longitud de los textos. Sobre este último punto, parece constituir un escollo para construir el sentido de lo que leen. Sin embargo, mientras que los alumnos con un alto rendimiento optan por leer la bibliografía propuesta, quienes poseen un rendimiento bajo apelan a resúmenes y carpetas hechas por terceros para simplificar la lectura —es decir, a la manera de fuentes secundarias - o para evadirla. Cabe señalar, que si bien esto último no ha sido descrito de manera análoga en la literatura revisada, resulta similar a los hallazgos de Iglesia y de Micheli (2009) sobre el empleo de resúmenes y apuntes de clase en lugar de los textos de las materias.

Solamente dos de los estudiantes con un alto rendimiento diferencian a nivel conceptual los textos que exponen y los que argumentan, ya que en la secundaria recibieron instrucción específica sobre esto (Arnoux et al., 2002; Padilla et al., 2015). En contraposición, únicamente los estudiantes y las estudiantes con un bajo rendimiento se centran en detalles superficiales (Iglesia y de Micheli, 2009) de los textos como el tamańo de la letra o el hecho de tener o no dibujos o paratextos.

Únicamente el estudiantado con calificaciones altas identifica dos formas de organización de las prácticas lectoras que resultan afines a las descritas desde la teoría como prácticas monológicas o en los extremos, y prácticas dialógicas, entrelazadas o de lectura compartida - una, que se centra en la identificación de información específica en los textos y la otra, que acentúa la relación entre lo propuesto por autores en diferentes textos, las explicaciones de los docentes y contextuar lo que se lee-.

Las primeras, se caracterizan por la preeminencia de la palabra del docente y tal como señalan estudios nacionales (Carlino, 2018a; Carlino, 2018b; Cartolari y Carlino, 2012; Villalonga Penna, 2017), sus explicaciones terminan siendo la fuente principal de información y la bibliografía no parece ser un medio para construir interpretaciones y apropiarse de los modos de decir en la disciplina de formación. Además, estas prácticas parecen no propiciar que los estudiantes realicen contribuciones significativas al tema trabajado (Cartolari y Carlino, 2012; Dysthe, 1996; Villalonga Penna, 2017; Wells, 2006). 
Las segundas, implican discusiones sobre lo leído (Carlino, 2018a; Carlino, 2018b; Cartolari et al., 2013; Braidot et al., 2012; Natale y Stagnaro, 2013; Villalonga Penna, 2017) en las que los docentes median los procesos lectores durante las clases e incentivan las interacciones para hablar sobre lo leído entre docentes y alumnos, y entre pares. Esto generaría zonas de intertextualidad entre los intercambios orales en el aula y el discurso escrito de la bibliografía disciplinar. Son interacciones en el aula en torno a los textos disciplinares que permitirían el acceso de los estudiantes a las formas de leer y comprender en disciplinas específicas (Cartolari y Carlino, 2012; Carlino et al., 2013; Dysthe, 1996; Villalonga Penna, 2017; Wells, 2006). A este respecto, valoran la segunda forma de enseńanza de manera positiva.

Además, el trabajo en clases prácticas, basado en preguntas que deben ser respondidas a partir de la lectura, resulta afín a los hallazgos de estudios nacionales (Carlino et al., 2013; Iglesia y de Micheli, 2009). Algunas, podrían responderse desde varios autores y entrelazando lo leído con las explicaciones del docente, lo cual daría cuenta de la necesidad de entrelazar o trabajar a partir de múltiples discursos o voces en torno a un tema (Carlino, 2017a; Carlino, 2018a; Carlino, 2018b; Carlino et al., 2017; Carlino et al., 2013; Cartolari y Carlino, 2011; Dysthe, 1996; Dysthe, Samara y Westrheim, 2006; Villalonga Penna, 2017; Wells, 2006). Otras preguntas, se responderían con partes específicas de los textos, lo cual implicaría contemplar solo una voz y por el tipo de secuencia planteada (el docente da una consiga, los alumnos responden y socializan, el docente evalúa), podría indicar que la lectura es una herramienta periférica (Carlino et al., 2013; Cartolari y Carlino, 2011; Dysthe, 1996; Dysthe et al., 2006; Wells, 2006).

La incongruencia percibida entre ambas formas de lectura parece generar incertidumbre en los estudiantes quienes declaran no poder terminar de comprender cuáles son las expectativas de los docentes de esta comunidad disciplinar, lo cual resulta coincidente con lo indicado por Lillis (1999) acerca de las discrepancias que pueden derivarse de la distancia entre las expectativas de los docentes y en qué medida estas son comprendidas o no por lo estudiantes. Sin embargo, a diferencia de lo que plantea la citada autora, este grupo de estudiantes estaría más familiarizado con las prácticas académicas de lectura que el grupo de los estudiantes con bajas calificaciones, ya que durante la escolaridad secundaria habrían construido conocimientos sobre las formas de leer y las clases de textos que se leen en la universidad. Si bien la incertidumbre de estos estudiantes podría implicar que para ellos la lectura es en cierta medida "una práctica del misterio", esta no lo sería en cuanto a las herramientas necesarias para comprender lo que se lee, sino solo en cuanto a cómo anticiparse a qué esperan los docentes de los estudiantes como lectores. En este caso, las prácticas de lectura y la comprensión de las normas y formas que estas adoptan, parecen ser vistas por los estudiantes y las estudiantes como algo problemáticas, aunque tengan conocimientos construidos en el nivel educativo anterior, lo cual se relaciona con los planteamientos de la citada autora.

En el caso del estudiantado que ha tenido bajas calificaciones, solo aluden a prácticas lectoras con base en la resolución de guías de estudio, tanto en el secundario como en la universidad. Sin embargo, plantean diferencias entre las mismas: las guías de estudio del secundario muchas veces resultaban estar resueltas o sus preguntas se respondían con fragmentos explícitos de un texto y, las de la universidad, se responden con diferentes partes de un texto o con diferentes textos. $\mathrm{Al}$ respecto, valoran negativamente la presencia de múltiples fuentes textuales, lo cual podría situarse en parte, en la misma línea que aquellos desarrollos acerca de las prácticas monológicas de lectura (Cartolari y Carlino, 2011; Iglesia y de Micheli, 2009) ya que el único discurso valorado es el del "resumen" realizado por el docente o por un tercero desconocido.

El trabajo en clases prácticas, a partir de preguntas a ser respondidas con base en la lectura, resulta similar al de estudios nacionales (Carlino et al., 2013; Iglesia y de Micheli, 2009; Villalonga Penna, 2017). Pero este grupo de estudiantes no puede realizar la relación de intertextualidad para responder algunos de los interrogantes, y los docentes parecen no ayudarlos de manera explícita a construir dichas relaciones. Las preguntas que se responderían con partes específicas de los textos y contemplando solo una voz y el tipo de 
secuencia planteada (el docente da una consiga, los alumnos responden y socializan, el docente evalúa), podría indicar que la lectura es una herramienta periférica (Carlino2013; Cartolari y Carlino, 2011; Dysthe, 1996; Dysthe et al., 2006; Wells, 2006). Cabe señalar que este tipo de práctica resulta familiar para estos estudiantes ya que las prácticas lectoras del nivel educativo anterior serían afines.

En este caso, los estudiantes solo describen con claridad una forma de organización de las prácticas lectoras que sería más de tipo monológica o en los extremos. Además, por la forma en que describen los textos que leen pareciera ser que están menos familiarizados con este aspecto de las convenciones de la lectura académica que el grupo anterior. Para estos alumnos la lectura podría ser una "práctica institucional del misterio" (Lillis, 1999, p. 127) ya que su bagaje anterior no les permite comprender las expectativas docentes en torno, por ejemplo, a cómo responder las guías de trabajo - que no son entregadas resueltas por los docentes y que no contienen preguntas cuyas respuestas se encuentran con facilidad en los textos- o a qué es lo que los docentes esperan de ellos como lectores académicos y no del secundario. Siguiendo a Collier (2001), estos estudiantes podrían haber percibido que lograban cumplir con las exigencias docentes en el secundario, pero en la universidad parecen percibirse como poco habilitados para leer y para comprender las demandas y adecuarse a las exigencias - lo cual se vería reflejado en su desempeño y en sus declaraciones-. Cabría preguntar si estas condiciones podrían llevarlos a desalentarse y/o frustrarse y abandonar los estudios superiores (Lea y Street, 1998).

En suma, el estudiantado con calificaciones altas tiende a percibir continuidades entre las prácticas lectoras del secundario y las de la universidad, y el que tiene calificaciones bajas, una incongruencia entre las mismas, lo cual se sitúa en la misma línea de los desarrollos internacionales y nacionales (Barton, Hamilton e Ivanic, 2000; Blommaert, Street, Turner y Scott, 2007; Carlino, 2019; Carlino, 2017b; Lillis y Scott, 2007). En consonancia con los hallazgos de estudios nacionales (Carlino y Estienne, 2004; Fernández y Carlino, 2010), tanto las similitudes como las distancias entre las prácticas lectoras de ambos niveles educativos se vinculan con la cantidad a leer, con la clase de textos a leer y con cómo se lee. En este sentido, puede verse cómo las experiencias lectoras construidas en el nivel secundario pueden facilitar u obstaculizar el aprendizaje de las prácticas lectoras disciplinares académicas (Collier y Morgan, 2000). Además, estos hallazgos muestran, en la misma dirección que Lillis (1999), cuáles son algunas de las condiciones de acceso y de permanencia en la educación superior en esta comunidad disciplinar en particular, y que no basta con que los alumnos asistan a clase, sino que es necesario que puedan participar de forma reflexiva en las prácticas de lectura propias de este nivel.

\section{Conclusiones}

Este estudio se centró en cómo conciben las prácticas lectoras académicas los alumnos del primer año de Psicología de una universidad de gestión estatal, y por ello, buscó describir dichas perspectivas. Los hallazgos dan a conocer que el estudiantado que tiene calificaciones altas tiende a percibir continuidades entre las prácticas lectoras del secundario y las de la universidad, y el que tiene calificaciones bajas, una incongruencia entre las mismas. Tanto las similitudes como las distancias entre las prácticas lectoras de ambos niveles educativos se vinculan con la cantidad a leer, la clase de textos y con cómo se lee. Estos resultados permiten entrever las diferencias derivadas de la escolaridad de base y la necesidad de abordar en las aulas universitarias la enseñanza de la lectura disciplinar en cada asignatura.

No puede dejarse de mencionar el alcance de nuestra investigación. Al tratarse de un estudio de carácter cualitativo en el que se toma solo el primer año de una carrera universitaria específica, los resultados no pueden generalizarse a toda la comunidad académica ni a todo el alumnado de los diferentes años de esta comunidad académica y disciplinar. Lo que puede resultar factible, es retomar los resultados y conclusiones de este estudio para pensar en casos análogos o similares. Por tratarse de un estudio de corte exploratorio y descriptivo, las categorías construidas debieran ser refinadas y profundizadas. 
Estudios posteriores podrían centrarse en una serie de interrogantes que se desprenden de nuestra investigación: ¿cómo se relacionan las perspectivas acerca de la lectura de estos estudiantes con las perspectivas acerca de la lectura de los docentes de esta comunidad disciplinar?, ¿cómo se relacionan las formas de concebir la lectura que poseen estudiantes y docentes con las prácticas lectoras desarrolladas en contextos áulicos?, ¿qué clases de textos leen los estudiantes en esta comunidad en particular?, ¿qué se hace con estos textos en las aulas universitarias? Siguiendo a Barton et al., (2000) sería útil contemplar un triángulo que incluya las prácticas de lectura del educador, las prácticas de los estudiantes y las prácticas letradas avaladas institucionalmente, ya que las mismas son eventos culturales que hacen necesaria una reflexión.

\section{Referencias}

Arnoux, E., Di Stefano, M. y Pereira, C. (2002). La lectura y la escritura en la universidad. Buenos Aires: Eudeba.

Barton, D., Hamilton, M. \& Ivanic, R. (2000). Situated Literacies. Reading and writing in context. Londres: Routledge, Taylor \& Francis Group.

Bigi, E., Chacón, E. y García, M. (2013). ¿Cuáles textos académicos leen los estudiantes universitarios en educación? Legenda, 17(17), 127-145.

Blommaert, J., Street, B., Turner, J. \& Scott, M. (2007). Academic literacies -what have we achieved and where to from here? Journal of applied linguistics, 4(1), 137-148.

Braidot, N., Moyano, E., Natale, L. y Roitter, S. (2012). Enseñanza de la lectura y la escritura como política institucional a lo largo de las carreras de ingeniería del IDEI-UNGS. En: Trabajos Completos, VI Congreso Argentino de Enseñanza de la Ingeniería (CAEDI), Salta, septiembre de 2012.

Carlino, P., Iglesia, P. y Laxalt, I. (2013). Qué dicen los docentes que hacen cuando los alumnos no comprenden lo que leen. Trabajo presentado en el XII Congreso Latinoamericano para el Desarrollo de la Lectura y la Escritura. IV Foro Iberoamericano de Literacidad y Aprendizaje.
Benemérita Universidad Autónoma de Puebla, Mexico, julio de 2013.

Carlino, P., Cordero, G., Roni, C., Rosli, N. (2017). Enseñar disciplinas con lectura y escritura: ¿qué interacciones se promueven y cuáles resultan epistémicas? Trabajo presentado en Writing Research Across Borders IV, Bogotá, febrero de 2017.

Carlino, P. (2017a). Aprender en la universidad: ¿qué diferencia hace si se lee, se escribe y se habla sobre lo leído y escrito? Trabajo presentado en el VIII Encuentro "Experiencias significativas en lectura y escritura". Red de Lectura y Escritura en Educación Superior-Redlees, Nodo Universidad del Valle, organizado por la Vicerrectoría Académica de la Universidad del Valle, la Dirección de Autoevaluación y Calidad Académica-DACA, el Programa para el Éxito Académico y el Grupo de investigación Leer, escribir y pensar, Cali, febrero de 2017.

Carlino, P. (2017b). Leer y escribir en las ciencias sociales en universidades argentinas. Enunciación, 22(1), 110-124. Disponible en https:// doi.org/10.14483/22486798.12350

Carlino, P. (2018a). Dos variantes de la alfabetización académica: cuando se entrelazan la lectura y la escritura en las materias. Signo y Pensamiento, 36(71), 16-32. Disponible en https:// doi.org/10.11144/Javeriana.syp36-71.dvaa

Carlino, P. (2018b). Leer y escribir para aprender a través del currículo en la educación superior: Investigaciones del GICEOLEM con variada aproximación metodológica. Trabajo presentado en el $30^{\circ}$ Aniversario del Centro de Investigaciones Educativas, San Juan, Universidad de Puerto Rico, mayo de 2018.

Carlino, P. (2019a). Escribir, leer y aprender en la universidad. Una introducción a la alfabetización académica. Buenos Aires: Fondo de Cultura Económica.

Carlino, P. (2019b). Dos variantes de la alfabetización académica. Trabajo presentado en el $I X$ Congreso Internacional de la Cátedra UNESCO para el Mejoramiento de la Calidad y Equidad de la Educación en América Latina, con base en la Lectura y la Escritura. Cuenca, julio de 2018. 
Cartolari, M. y Carlino, P. (2012). Leer y tomar apuntes para aprender en la formación docente: un estudio exploratorio. Magis. Revista Internacional de Investigación en Educación, 4(7), 67-86.

Cartolari, M. y Carlino, P. (2016). Hacerle lugar o no a la lectura en aulas del nivel superior: experiencias contrastantes en dos materias de un Profesorado en Historia. En G. Bañales Faz, M. Castelló Badía y N. Vega López (comps.) Enseñar a leer y a escribir en la educación superior (pp. 160-180). México DF: SM México y Univ. Autónoma de Tamaulipas.

Collier, P. (2001). A differentiated model of role identity acquisition. Symbolic interaction, 24 (2), 217-235. Disponible en http://www.jstor.org/ stable/10.1525/si.2001.24.2.217

Collier, P. J. \& Morgan, D. L. (2008). Is that paper really due today?: differences in first-generation and traditional college students' understandings of faculty expectations. Higher Education, 55(4), 425-446.

Dysthe, O. (1996). The Multivoiced Classroom Interactions of Writing and Classroom Discourse. Written communication, 13(3), 385-425.

Dysthe, O., Samara, A. \& Westrheim, K. (2006). Multivoiced supervision of Master's students: a case study of alternative supervision practices in higher education. Studies in Higher education, 31(03), 299-318.

Fernández, G. y Carlino, P. (2010). ¿En qué se diferencian las prácticas de lectura y escritura de la universidad y las de la escuela secundaria? Lectura y Vida, 31(3), 6-19.

Giudice, J., Godoy, M. y Moyano, E. I. (2016). Prácticas de lectura y escritura en el marco de la enseñanza de la Psicología: avances de una investigación interdisciplinaria. Revista mexicana de investigación educativa, 21(69), 501-526.

Godoy, M. y Giudice, J. (2015). Problemática de los docentes en relación a las prácticas de lectura y escritura de sus alumnos universitarios y una modalidad de abordaje desde la gestión. Ponencia presentada en el Congreso Nacional Catedra UNESCO para el Mejoramiento de la calidad y
Equidad de la Educación en América Latina, con base en la Lectura y en la Escritura. Rosario. 22, 23 y 24 de septiembre de 2014.

Heinemann, W. \& Viehweger, D. (1991). Textlinguistik: eine Einführung. Berlin: Walter de Gruyter.

Hermerschmidt, M. (1999). Foregrounding background in academic learning. Students writing in the university: cultural and epistemological issues. Amsterdam, 1, 6-16.

Hernández, R., Fernández, C. y Baptista, P. (2008). Metodología de la Investigación. México: McGraw Hill.

Iglesia, P. y De Micheli, A. (2009). Leer textos de biología en el primer año de la universidad: ¿es un saber construído o una práctica a enseñar? Enseñanza de las ciencias: revista de investigación y experiencias didácticas, (Extra), 817-820.

Lea, M. R. \& Street, B. (1998). Student writing in higher education: An academic literacies approach. Studies in higher education, 23(2), 157-172.

Lillis, T. (1999). Whose common sense. Essayist literacy and the institutional practice of mystery. 127-140.

Lillis, T. \& Scott, M. (2007). Defining academic literacies research: issues of epistemology, ideology and strategy. Journal of applied linguistics, 4, 5-32.

Natale, L. y Stagnaro, D. (2013). Alfabetización profesional durante la carrera universitaria: entre la universidad y la empresa. Itinerarios educativos, 7, 11-28.

Padilla, C. (2017). Desafios epistêmicos e argumentativos na escrita da Pós-graduação: gêneros científico-acadêmicos e trajetórias de mestrandos e doutorandos. EID\&A, 15, 270 - 295

Padilla, C., Douglas, S. y López, E. (2007). Yo expongo. Taller de prácticas de comprensión y producción de textos expositivos. Córdoba: Comunicarte.

Padilla, C., Douglas, S. y López, E. (2011). Yo argumento. Taller de prácticas de comprensión y producción de textos argumentativos. Córdoba: Comunicarte.

Padilla, C., Douglas, S., y López, E. (2015). Investigar, leer y escribir en la universidad: recorridos de estudiantes ingresantes de Humanidades. Enunciación, 19(1), 65-80. 
Villalonga, M. M. (2015). ¿Qué clases de textos leen en primer año los estudiantes de Psicología? Material de Estudio y perspectivas docentes. En B. Gabbiani y V. Orlando (Coord.) Escritura, lectura y argumentación en contextos educativos del Río de la Plata (pp. 45-66). Montevideo: Universidad de la República.

Villalonga, M. M. y Hael, M. V. (2016). Leer en Psicología: ¿qué elementos de los textos académicos reconocen los estudiantes de un curso avanzado? Revista Iberoamericana de Educación Superior, UNAM, IISUE, 20(7), 157-171.

Villalonga, M. M. y Padilla, C. (2016). ¿Qué textos leen en primer año los estudiantes de Psicología? Organización del material bibliográfico, elementos para contextuarlo, clases de textos y tipos discursivos predominantes. Educacao e Pesquisa, 43(2), 515-533.
Villalonga, M. M., Padilla, C., Carreras, M.P., Carreras, M.A. (2019). Leer en el primer año de Psicología: tipos discursivos e inferencias. Revista Iberoamericana de Educación, 80(2), 81-103.

Wells, G. (2006). Monologic and dialogic discourses as mediators of education. Research in the Teaching of English, 41(2), 168-175. 